\title{
Shape and uneven heating correction for NDT on cylinders by thermal methods
}

\author{
by J.F. Pelletier ${ }^{1}$, E. Grinzato ${ }^{2}$, R. Dessi ${ }^{2}$, X. Maldague ${ }^{1}$ \\ ${ }^{1}$ Université Laval, Cité Universitaire, Québec, Canada, GIK $7 P 4{ }^{2}$ Consiglio Nazionale delle Ricerche - ITEF, \\ corso Stati Uniti 4, 35127 Padova, Italy.
}

\begin{abstract}
Use of the 'Shape from Heating technique' for the non-destructive detection of delaminations on cylinders made of composite materials is presented. A particular solution of the general technique is described to compensate the thermogram for both effects of non planar shape and non uniformity of the heat source. The processing is relatively fast since symmetry of the target is used. The proposed solution, even if not general, can nevertheless be applied to many industrial problems of interest.
\end{abstract}

\section{Introduction}

The Non Destructive Testing (NDT) of materials and the detection of defects is a growing field of interest for dynamic infrared thermography. Unfortunately the complex shape of targets creates a great difficulty for the quantitative analysis of thermograms. This is particularly true when an automatic process is used or when results are evaluated by unspecialised operators.

A technique known as Shape from Heating $[1,2]$ applied to transient IR thermography allows the determination of geometric features of the inspected object. The orientation and the relative distance of each surface element is obtained by processing of a single thermogram, recorded immediately after irradiation of the target. This technique derives from the Shape from Shading method [3] developed for robot vision in the visible band. The knowledge of the shape of the target allows the correction of the measured radiometric intensity on thermograms for geometrical effects. This paper describes a simplified algorithm, derived from the general one, in the case of cylindrical objects (a common application encountered on the plant floor).

The objects considered for the reported experiments are cylinders used as electrical insulators in power plants. In this particular case, straightforward application of transient thermal methods for the NDT of defects $[4,5]$ is limited to a region close to the cylinder axis. Unfortunately, if the surface is glossy, as in the present case, this area is normally very disturbed by reflections of the impinging radiation. A further problem encountered is the presence of a gradient along the cylinder axis created by the uneven heating of the surface. These drawbacks could be overcome by a double application of the one-dimension version of the 'Shape from Heating' approach.

In order to simplify as much as possible the algorithm, the number of variables is limited by freezing the experimental conditions and performing a preliminary calibration phase. Therefore the experimental set-up must be characterised before extraction of the object geometry. The correction procedure is applied next.

In the following, the correction algorithm is first applied on a plastic cylinder for calibration purposes. In section 5, experimental results are reported on a real target.

\section{Description of the algorithm}

The symmetry of the cylinder allows adoption of the 1D version of 'Shape from Heating'. Fig. 1 shows a thermogram obtained after heating a plastic pipe made of PVC and black painted, except for two triangular markers. Choosing the axis of the cylinder as $y$, the geometry changes only along the cylinder cross section ( $x$ axis). In such a way, the cylinder image is decomposed in a series of parallel profiles. As a consequence, the analysis of only one profile is needed to obtain the geometric description of the target.

According to $[1,2]$, recovering of the geometry could be done using a linear or non-linear model. The linear model considers the radiometric intensity changes due to the depth of the object. In the non-linear case, the intensity varying is mainly due to the local variation in the surface orientation. In our case, because the object's segments have a curvature different from zero, the non-linear model applies to the $x$ axis while correction along the $y$ axis for the variation of the irradiation, combined with a tilting of the cylinder requires a linear model. 


\subsection{Procedure for the extraction of the best profile}

The first task is the extraction of a suitable crossed profile in the thermogram from which the cylinder geometry is recovered. Eq.(1) describes the temperature increase on the object (Tx-To) due to the irradiation, according to the non-linear model. In fact it depends only on the local orientation $(\theta)$ of the surface [6].

$$
\left(T_{x}-T_{o}\right)=\left(T_{m}-T_{0}\right) \cos \theta
$$

Here $T_{0}$ is the initial temperature and $T_{m}$ the temperature on a reference point. It is convenient to use as reference the point, the maximum temperature belonging to the crossed profile. In such a way we may consider its orientation equals to zero, that is, normal to the heating source. All others orientations may be derived relatively to this reference point.

The average absolute error for each crossed profile is computed as the difference in the orientation if compared with an ideal cylinder of the same diameter. Finally the profile along the cylinder axis with the minimum error is chosen for temperature correction.

Once the orientation of the surface is known, depth variations $(d z)$ of each pixel are calculated with eq.(2) starting from the projected distance $(d x)$ along the $x$ axis, measured on the image.

$$
d_{z}=d_{x} \tan \left(\frac{\theta}{2}\right)
$$

A preliminary calibration stage allows the definition of the scale factor, by means of a marker of known size. In the case of the orthogonal projection, this calibration is valid for the $x$ and $y$ axis as well. In the general case, the perspective correction requires the use of a suitable calibration image and also to compute the $d x$ and $d y$ for all pixels. This approach imposes a limit to the procedure since the experimental rig must not change in time (otherwise another calibration is needed).

In fig. 2 the surface orientation and in fig. 3 the depth for such a profile are shown for the calibration cylinder, in comparison with the ideal one.

\section{Characterisation of the experimental system and noise filtering}

The targets are heated by a set of flash tubes. A thermographic device records sequences of thermograms at a frame rate of 15 or $30 \mathrm{~Hz}$. The object and flashes are put inside a reflective cabinet. Of course this experimental rig is just an example and other configurations are possible (especially for the industrial deployment of the procedure).

In practice, an even heating of the target is fairly impossible. Therefore the original method, requiring an uniform heating with parallel rays, is to be modified. For this purpose the distribution of the irradiation is quantified and a correction for the impinging energy is applied to each pixel belonging to the cylinder, as described in section 4.

An important step for the system characterisation is the evaluation of the noise level present in the data. The adopted parameter is the standard deviation $(\sigma)$, computed on the map of the noise [4]. This map is obtained as the difference between two thermograms recorded before the heating at the highest speed (time step of $33 \mathrm{~ms}$ in our case). The test was repeated three times giving as results: $\sigma=1.3649,1.2849$ and 1.2049. The mean value was adopted.

\subsection{Noise filtering}

The noise was filtered by approximating each profile with a series of small segments given by a polynomial function of the first order [7]. The coefficients $A$ and $B$ are computed at point $x$ using the least square method. In this way, the filtered values are the central points of each segment. The length of the segment is chosen longer than the standard deviation of the noise. in our case this implies two pixels for each side of the considered one.

An alternative method computes the intensity of the smoothed profile by a convolution with the two following kernels $K[A]=1 / 10 *[2,1,0,-1,-2]$ and $K[B]=1 / 5 *[1,1,1,1,1]$. A running window sized at 5 pixel width was used according to the following formula:

$$
T_{x}=A \cdot x+B
$$

Fig. 4 shows an example of noise filtering for the calibration cylinder as compared with the original cross section profile. 


\section{Correction of thermograms}

\subsection{Uneven heating correction}

The first step refers to the correction of the uneven distribution of the stimulating energy on the surface. An homogeneous flat screen placed behind the target gives a map of the energy distribution. Looking at the temperature variation on the background of fig.1, the thermal gradient due to the source is found principally oriented along the $y$ axis. Applying the non-linear correction independently for each crossed section profiles frees to take into account the different irradiation along the cylinder axis. For this purpose the scene is scanned by columns and the slope of the intensity is computed by fitting each column with a first order function. Therefore, using as reference the best profile extracted $\left(y_{0}\right)$, each pixel temperature is corrected by its distance from the reference crossed profile according to eq.(4)

$$
T_{c}=\left[\Delta T\left(y_{0}-y\right)\right]+T
$$

An effective procedure for the enhancement of temperature patterns due to a possible defect inside the target is to subtract the corrected thermogram to suppress most of geometric and uneven heating effects with a theoretical model of the cylinder. Such a model is obtained repeating the best profile yo as many times as the number of crossed profiles. Fig. 5 shows the map of the errors between experimental data and the theoretical model for the calibration cylinder. In this case, no defect exists but the two markers are clearly visible in a more or less flat background.

The final correction for the uneven heating processes as follows:

- a threshold is chosen and applied to the error image;

- the resulting binary image is used as a mask and each point having a value lower than the threshold is replaced in the original image by the value corresponding to the best profile. If the error is higher than the threshold, the value is let unchanged.

Using this procedure, a corrected image is obtained with defects clearly visible, as fig.6 shows. It is important to apply the correction for the uneven irradiation from the very early stage of heating, when the lateral diffusion of heat may be neglected.

Now, the correction for the cylinder shape is next applied.

\subsection{Correction for shape}

Knowing the orientation of each elementary area of the cylinder, eq. (1) is applied to the original temperature increase. After this correction, each pixel stretches its intensity to a level equal to that reached by a pixel perpendicularly facing the source.

The procedure is repeated for the whole image. Fig.7 shows the result of the procedure.

\section{Delaminations detection on a fibreglass cylinder}

A specimen of a composite fibreglass cylinder was prepared with artificial delaminations. The sample is made of two layers: a fibreglass poltruted core and the polyester fibres layer immersed in a matrix resin. Delamination defects of thickness of tens of microns could be in between. The specimen was blackened with a diffusing wash-removable painting to have a constant angular dependence on emissivity.

The thermal camera used was an Agema $900 \mathrm{LW}$ and the heating device was a set of three flash lamps with a pulse duration of $10 \mathrm{~ms}$ (delivering an energy of $3 \mathrm{~kJ}$ each). The thermographic system and heat sources were synchronised together. Fig. 8 shows the third raw thermogram grabbed $130 \mathrm{~ms}$ from the start of heating.

An automatic processing of data extracts defects, according to the following procedure [8] :

- a sequence of thermograms was recorded and the first image was taken before the start of heating;

- for all pixels belonging to images the temperature increase was immediately computed;

- the experimental rig was not changed after the preliminary calibration stage, so the parameter $d x$ an $d y$ were retained from this calibration.

Fig. 9 shows the delaminated area clearly visible on the top, although three linear false alarms can be seen as well, probably due to a non uniformity in the paint thickness. These artefacts and others spikes are filtered in a post processing phase using a morphological filter. 


\section{http://dx.doi.org/10.21611/qirt.1996.043}

The actual NDT algorithm is out of the scope of this paper. Nevertheless, the proposed procedure outlines are as follow: if the amplitude of the thermal contrast [5] produced by defects is quite high, a trivial threshold approach allows detection. A minimum signal to noise ratio of two has been evaluated as general rule for detectability.

In the case of a slowly varying contrast, attention must be paid to possible errors in defect sizing induced by the thresholding procedure. A more sophisticated algorithm must be adopted for this purpose. In any case, various algorithms may be applied to the error image to optimize the detection phase, depending on particular requirements.

\section{Conclusions}

In this paper we proposed a method to correct thermal images of a cylindrical object both for unplanar geometry and uneven heating. The main advantage of this procedure is its capability to correct the thermogram for shape effects using only the best crossed profile of the cylinder.

The main obstacle encountered was the uneven heating, more than the shape. Probably, a more complex function than a linear interpolation is needed if using spot heaters (as flash lamps). In any case, it is very important to achieve a relatively constant irradiation. Nevertheless, the results given by the simplified procedure for the heating correction are satisfying.

This procedure is based on certain constrains but they may be loosen if required. An interesting point emerged from experiments : this is the possibility to process more complex images with different cylindrical objects present simultaneously. In this case, each cylinder is processed individually and the geometric correction applies only in one direction.

Finally, it must be noted that such a procedure is suitable only for the defect identification because only relative values between defect and sound areas are available after processing. $A$ new procedure for the characterisation of defects on cylinder is now planned.

\section{Acknowledgements}

Authors wish to acknowledge the contributions of the Magrini Galileo S.p.a., Schneider group, for the technical support and manufacturing of specimens and of the Natural Sciences and Engineering Research Council of Canada, the Ministère des Affaires Internationales du Québec, the Ministère de l'Éducation du Québec, the Institute for Robotics and Intelligent Systems (Université Laval's node) for their financial support.

\section{REFERENCES}

[1] Laurendeau (D.) - Shape Reconstruction from a Single Thermal Image. Optical Engineering, Vol. 34, $\mathrm{n}^{\circ}$. 1, pp. 154-159, 1995.

[2] Pelletier (J. F.), Maldague (X.) - Infrared Thermography : Nonplanar Inspection". SPIE Thermosense

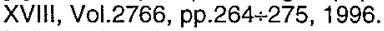

[3] Horn (B.K.) - Robot vision the MIT press, 1982.

[4] Maldague (X.) - Nondestructive Evaluation of Materials by Infrared Thermography. Springer-Verlag, London, 1993.

[5] Vavilov (V.), Grinzato (E.), Bison (P.G.), Marinetti (S.), Bressan (C.) - Thermal Characterisation and Tomography of Carbon Fibre Reinforced Plastics Using Individual Identification Technique. Material Evaluation, pp.604-610, 1996.

[6] Maldague (X.), Barker (E.), Nouah (A.), Boisvert (E.), Dufort (B.), Fortin (L.), Laurendeau (D.) - On Method for Shape Correction and Reconstruction in Thermographic NDE. Advance in Signal Processing for Nondestructive Evaluation of Materials, NATO-ASI series, Vol. 262, pp. 209-224, Kluwer Academic Publishers, 1994.

[7] Pelletier (J.F.), Grinzato (E.), Dessì (R.), Maldague (X.) - Application du shape from shading pour la correction de la géométrie de cylindres de fibres de verre un vue de la détection dés delamination par thermographie infrarouge. Quaderno CNR-ITEF $\mathrm{n}^{\circ} .1 / 96$, Padova, 1996.

[8] Maldague (X.), Krapez (J. C.), Poussart (D.) - Thermographic Nondestructive Evaluation: An Algorithm for Automatic Defect Extraction in Infrared Images. IEEE Trans. on Systems, Man and Cybernetics, Vol. $20, n^{\circ} 3, p p .722-725,1990$. 

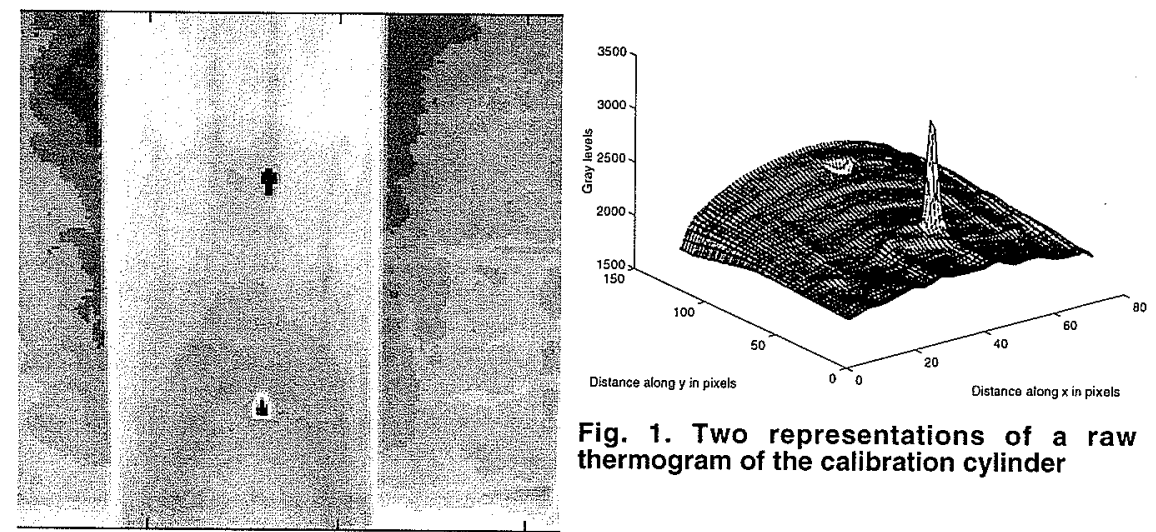

Fig. 1. Two representations of a raw thermogram of the calibration cylinder
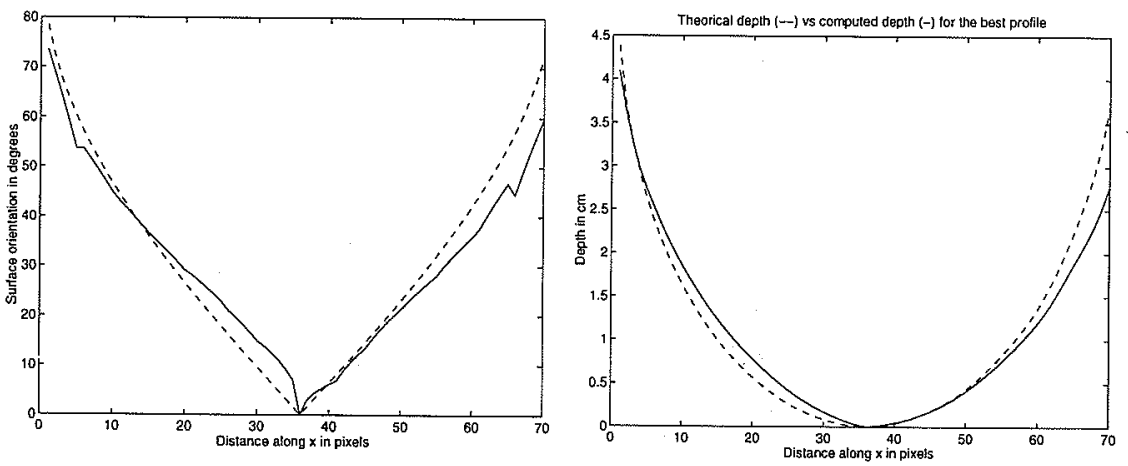

Fig. 2 . Surface orientation computed for the best profile compared with the ideal case

Fig. 3. Surface distance computed for the best profile compared with the ideal case
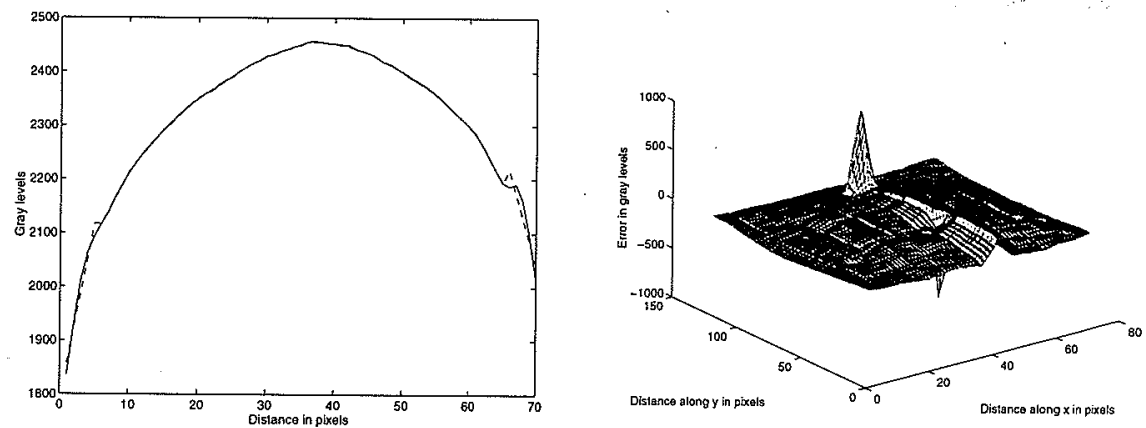

Fig. 4 . Original cross profile and result of the noise filtering (solid line)

Fig. 5. Error image for the calibration cylinder 


\section{http://dx.doi.org/10.21611/qirt.1996.043}

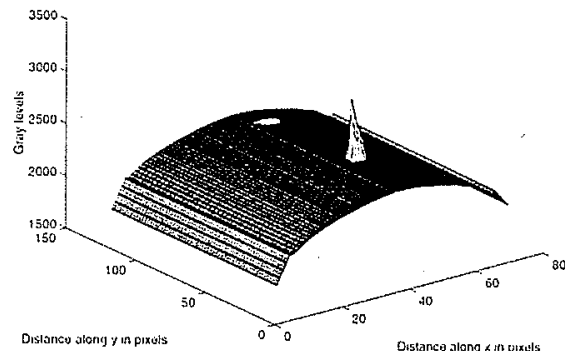

Fig. 6 . Correction for the uneven heating on the calibration cylinder

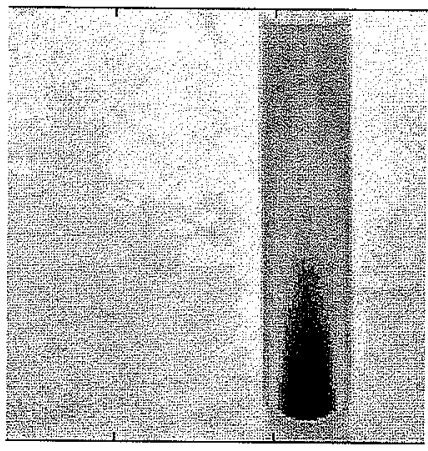

Fig. 8 . Row thermal image of the fibreglass cylinder after heating

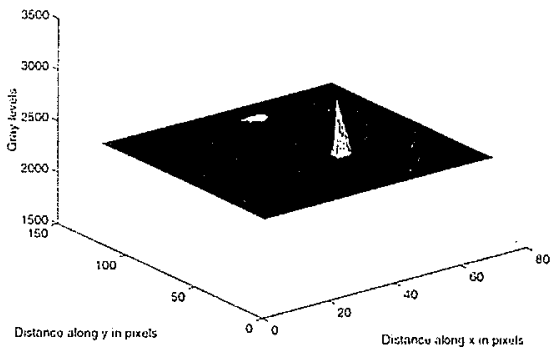

Fig. 7 . Fully corrected thermogram both for shape and heating

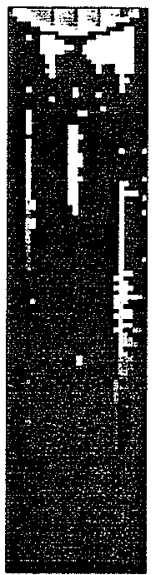

Fig. 9. Defect map on the corrected thermogram; only the cylinder is extracted and processed 